\title{
CIÊNCIA'NATURA
}

\section{Avaliação socioeconômica dos piscicultores do município de Porto Grande, Amapá, Brasil}

Socioeconomic evaluation of fish farmers in the municipality of Porto Grande, Amapá, Brazil

Netiê Izabel da Silva de Oliveira ${ }^{1}$, Alexandro Cezar Florentino ${ }^{2}$

${ }^{1}$ Centro Integrado de Formação Profissional em Pesca e Aquicultura do Amapá - CIFPA, Macapá - AP, Brasil.

${ }^{2}$ Universidade Federal do Amapá, Macapá - AP, Brasil.

\section{Resumo}

A caracterização do perfil socioeconômico é uma ferramenta essencial para diversos tipos de análises, quando se quer saber as relações dos processos sociais, a produção econômica sobre uma determinada área, as condições de vida de uma determinada comunidade ou grupo de pessoas, assim, como determinar o seu padrão de crescimento e desenvolvimento. O presente estudo teve objetivo avaliar o perfil socioeconômico de piscicultores do município de Porto Grande, Amapá, Brasil. Foram entrevistados dezoito piscicultores, como suporte metodológico, utilizou-se a pesquisa qualitativa e quantitativa, com aplicação de formulários, nestes estão expostas perguntas estruturadas e semiestruturadas. Com análise estatística englobando todas as etapas da estatística descritiva. Os principais resultados da investigação são a predominância do sexo masculino na atividade, a idade média de 48,07士 anos, em sua maioria os entrevistados são casados. Na escolaridade, houve predominância do nível fundamental incompleto. A piscicultura não é apresentada como renda exclusiva, precisando de complementações para suprir as necessidades das famílias, entre elas a agricultura. A partir desses resultados, foi possível analisar um leque de situações, que nunca foram discutidas em outros trabalhos científicos para essa região, o que enaltece a importância desse levantamento socioeconômico.

Palavras-chave: Piscicultura. Condições de vida. Frequência.

\section{Abstract}

The characterization of the socioeconomic profile isanessential tool for various types of analysis, whe nonewantsto know the relations of social processes, economic production over a givenarea, the living conditions of a particular community orgroup of people, and howto determine Its patternofgrowth and development. The present study aimed to evaluate the socioeconomic profile of fishfarmers in the city of Porto Grande, Amapá, Brazil. Eighteen fishfarmers wereinterviewed, as a methodological support, weused qualitative and quantitative research, with application of forms, in these are exposed structured and semi-structured questions. With statistical analysis encompassingallstages of descriptive statistics. The main research findings are the predominance of males in the activity, the mean age of $48.07 \pm$ years, the majority of respondents are married. In school, therewas a predominance of incomplete fundamental level. Fish farmingis not presented as exclusive income, requiring supplementation to meet the needs of families, including agriculture. From the seresults, it was possibleto analyze a range of situations that were never discussed in o thers cientific studies for this region, which praises the importance of this socioeconomic survey. 


\section{Introdução}

A procura por alimentos saudáveis e ricos em proteínas transformam o pescado como principal componente na dieta alimentar da população mundial. Este fato provavelmente contribui para que a maioria das áreas de pesca já se apresentem com ápice de extração. Assim, devido a esse colapso da sustentabilidade da atividade de pesca que começou a se sobressair à aquicultura, principalmente a criação de peixes no Brasil, busca pela alternativa de obtenção de pescado por meio de criação em aquicultura já é praticada há muito tempo, o que sugere que ela seja exercida mesmo antes do descobrimento do país, a diferença que atualmente essa prática possui finalidade econômica (BOEGER e BORGHETTI, 2008).

A piscicultura surgiu como alternativa para suprir a demanda de peixes, seja pelo aumento demográfico ou por requerimentos proteicos. Esta tende a se tornar uma interessante alternativa para as comunidades ribeirinhas, pescadores e assentamentos rurais, diante dos grandes processos exaustivos de extração da pesca extrativista, que contribuíram para a diminuição da oferta de peixes, um importante alimento rico em nutrientes (SOUZA, 2006; ARÊAS et al., 2014). Para diversas populações, incluindo aquela do município de Porto Grande, estado do Amapá.

O município de Porto Grande possui condições privilegiadas para o desenvolvimento da piscicultura, devido sua disposição de malha hídrica (GAMA, 2008). O aparecimento desta atividade na região despertou o interesse das comunidades locais. E a atividade aquícola no município, foi aprovada pela LEI Municipal № 413 de 26 de Junho de 2014, dispondo sobre a Atividade de Aquicultura e de outras Providências.

A escolha da criação de peixes como atividade rentável para as famílias das comunidades de Porto Grande gerou a necessidade de ser feito um diagnóstico do perfil socioeconômico para identificar características na questão social e condições de vida dessas pessoas produtoras de peixes (piscicultores). A caracterização do perfil socioeconômico é uma ferramenta essencial para diversos tipos de análises, quando se quer saber as relações dos processos sociais, a produção econômica sobre uma determinada área, as condições de vida de uma determinada comunidade ou população, assim, como determinar o seu padrão de crescimento e desenvolvimento.

Através do perfil socioeconômico evidencia-se o papel das famílias dentro da sociedade, este viabiliza a compreensão dos mecanismos adotados para a sobrevivência dos componentes de uma família (IBGE, 2012). Assim, a Síntese de Indicadores Sociais - "Uma análise das condições de vida da população brasileira", reitera que a dinamização da captação em relação ao funcionamento da dinâmica dos grupos familiares torna-se a ferramenta essencial para identificar o perfil socioeconômico, principalmente o eixo condições de vida, possibilitando entender todos os aspectos que compõem a vida social (IBGE, 2012).

A análise das condições de vida dos brasileiros é possível através da mensuração das condições de habitação de cada família, isso engloba parâmetros que são distribuídos desde o acesso a sua residência, promoção de serviços públicos como saneamento, iluminação, conforto, estética da residência até a disponibilidade de água. Pois, a compreensão das condições de vida de cada família reflete a sua própria organização familiar e sua representatividade na sociedade (IBGE, 2012). Desta forma, este trabalho avalia o perfil socioeconômico dos piscicultores de Porto Grande no Estado do Amapá, Brasil, evidenciando vários aspectos relacionados as condições de vida, naturalidade, faixa etária, gênero, existência de bens duráveis e renda.

\section{Material e Métodos}

\section{1 Área de estudo}

O município de Porto Grande (Fig. 1) possui um território de $4.401,774$ km² de extensão o que equivale a 3,08\% do território amapaense, $0,051 \%$ do território brasileiro (IBGE, 2010). As áreas do município de Porto Grande que foram contempladas por esse estudo, são as que possuem atividade de piscicultura, que envolve as comunidades de Cupixi, Nova Canaã, Nova Colina, Munguba, Perimetral Norte, Colônia Agrícola do Matapi, Estrada de Ferro e Área Urbana do Município, e algumas pisciculturas mapeadas nos Quilômetros 126 km, 134 km, 135 km e 142 km. No município de Porto Grande segundo a Classificação climática de Köppen-Geigero clima predominante é o equatorial super-úmido (Am) (SUDAM, 1984; ECOTUMUCUMAQUE, 2010).

O município é banhando principalmente pela bacia hidrográfica do Rio Araguari, que possui sua nascente na Serra do Tumucumaque, e deságua em direção ao Atlântico e pelos rios Amapari, afluente do Araguari e Matapi e o Vila Nova. A magnitude desta Bacia, com aproximadamente $42 \mathrm{mil} \mathrm{km} \mathrm{km}^{2}$ de área total e vazão média de $1.200 \mathrm{~m}^{3} / \mathrm{s}$, apresenta um excepcional potencial energético (PPCDAP, 2009; ECOTUMUCUMAQUE, 2010).

\subsection{Coleta e análise de dados}

As entrevistas foram direcionadas com base no estabelecimento de um grau de interação com nossos alvos de estudo, estas estabelecem uma assimetria dentro do diálogo, visto que a parte interessada busca informações a respeito de um determinado acontecimento ou da natureza de um fato e a pessoa entrevista oferece a fonte dessas informações requeridas (GIL, 2008). 
Figura 1- Localização do Município de Porto Grande, Amapá, Brasil. Com ênfase (pontos vermelhos) nas propriedades visitadas onde ocorreu a aplicação dos formulários para este estudo.

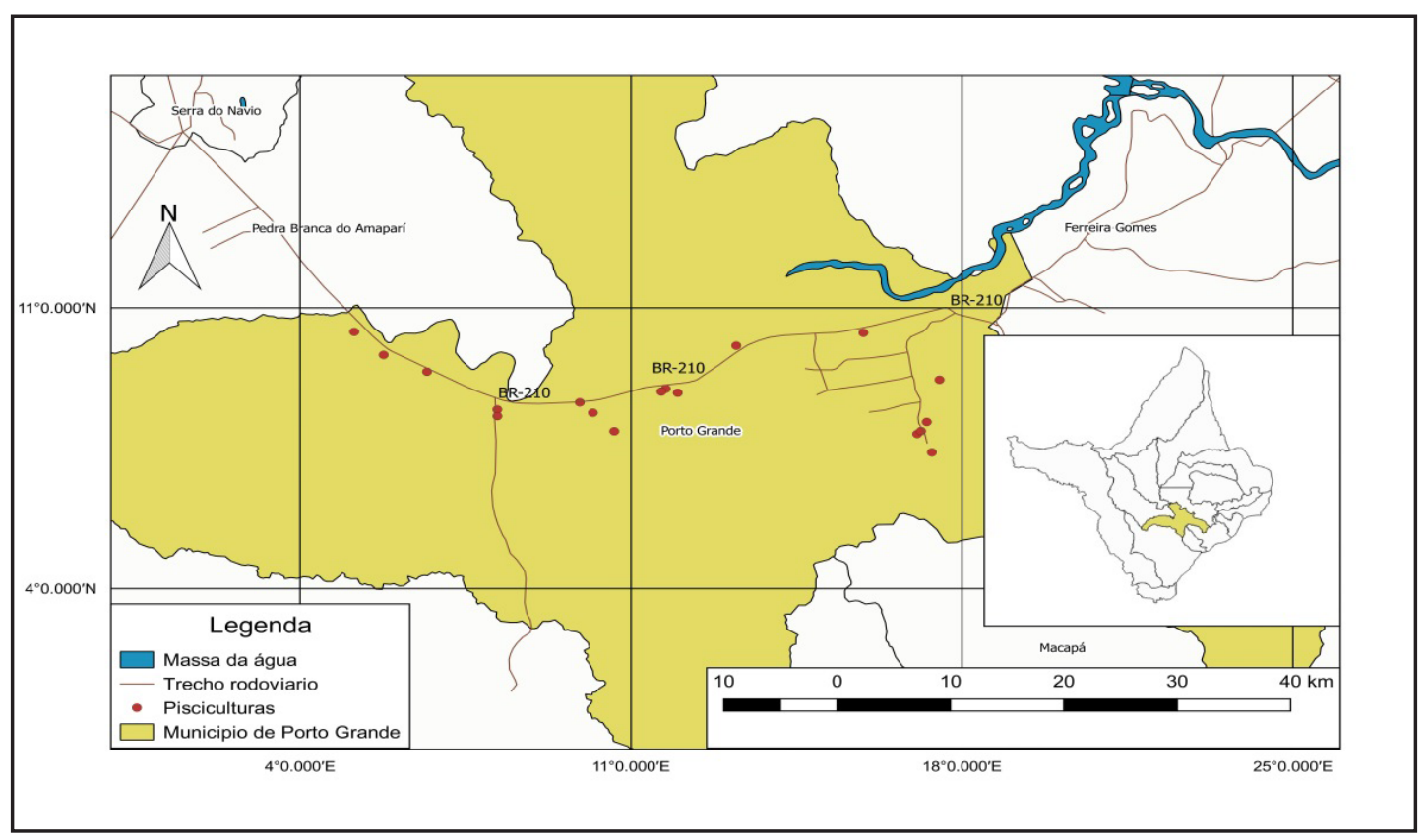

As entrevistas com auxílio de formulários estruturados com questões abertas e fechadas ocorreram no período de dezembro de 2015 a julho de 2016, foram aplicados para dezoito piscicultores.

Os dados foram analisados através da estatística descritiva, calculando todos os seus parâmetros, mínimo, máximo, moda, desvio padrão, média. Estabelecendo a frequência absoluta e frequência relativa, e quando necessária, a soma dessas frequências.

\section{Resultados e Discussões}

\subsection{Idade e gênero}

A amostragem obtida durante a aplicação dos formulários nas propriedades dos piscicultores, proporcionou a análise de diversas informações, entre elas, a faixa etária, compreendida com valores que vão inicialmente a 24 anos até aos 76 anos, distribuídas nos gênero feminino e masculino respectivamente. A idade média, para ambos os gênero, dos entrevistados foi de 48,07 anos.

A média de idade de 48 anos é evidenciada no IMEA (2014), na Região Norte de Mato Grosso. Neste mesmo trabalho, na Região Meio-Norte os piscicultores possuem idade média de 49 anos. Mostrando grande similaridade nos resultados deste estudo. Em Tabatinga, Nakauth et al. (2015) descrevem a piscicultura como uma atividade predominantemente masculina, composta por $67,86 \%$ dos produtores e com faixa etária em torno de 55 anos.

Em relação ao gênero, assim como os resultados expressados acima, o trabalho de Araújo (2015), identifica que todos os piscicultores entrevistados são do sexo masculino, correspondendo a 100\% do total. O autor justifica, pelo fato da função de ser uma atividade que exige bastante esforço físico, por este motivo é ocupada por pessoas do sexo masculino. O gênero masculino como mais frequente nessa atividade é descrito por Agboola (2011), no Estado de Osun, Nigéria; por Gomes et al. (2012) em Blumenau, Santa Catarina; por Gupta and Dey (2014) na cidade de Lumindo, Nagaon, Distrito de Assam, por Dubey et al. (2016) no delta Sundarban indiano. As investigações nas literaturas pressupõem que a idade média dos piscicultores não se difere em grande escala e o gênero masculino como hegemônico na criação de peixes torna-se comum para todas as regiões estudadas.

\subsection{Nível Educacional}

Os dados demonstram que, metade dos entrevistados não terminou o ensino fundamental, sendo $11 \%$ do sexo feminino e 39\% masculino. Somente 6\% não são escolarizados, ressalta-se que esta estimativa equivale a apenas um entrevistado, veja na Figura 2. 
Figura 2 - Escolaridade dos piscicultores de Porto Grande, Amapá, Brasil

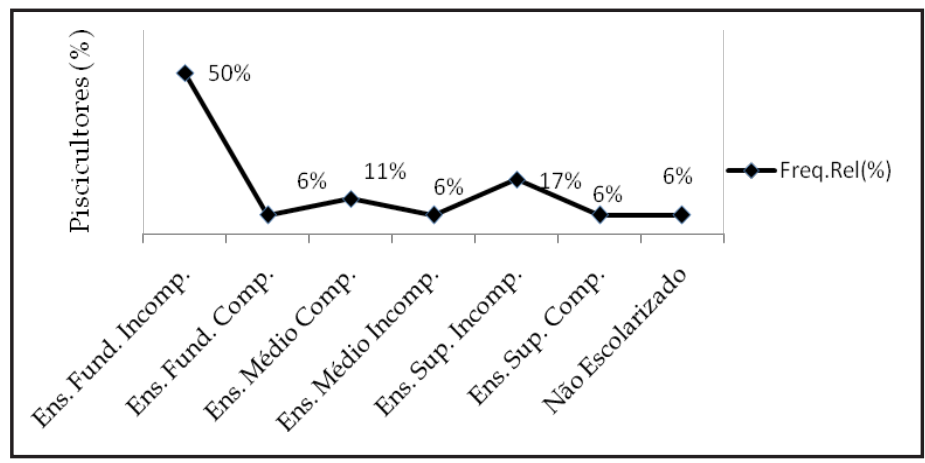

É através da educação formal que são oportunizadas a inserção profissional, o nível de frequência escolar do indivíduo possibilita a ascensão de oportunidades de emprego mais valorizadas economicamente. Segundo o IBGE (2012) a educação é um bem coletivo em si mesmo, essencial para a promoção da cidadania, apresentando um visível impacto nas condições gerais de vida da população, o que a torna cada vez mais imprescindível para a inserção social plena.

Sarah et al. (2013) estudando produtores rurais no município de Cruzeiro do Sul no Acre constataram que $60 \%$ dos piscicultores entrevistados não concluíram o Ensino Fundamental completo. Nakauth et al. (2015) estudando a caracterização da piscicultura em Tabatinga (AM), no período de agosto a dezembro de 2012 contatou que a escolaridade predominante foi o ensino fundamental incompleto, representado por $68 \%$ do total dos entrevistados. Dados levantados no Diagnóstico da Piscicultura em Mato Grosso pelo IMEA (2014) demonstram que 22,08\% dos entrevistados também possuem o Ensino Fundamental Incompleto, e apenas 16,02\% possuem o Ensino Fundamental Completo. Tais resultados indicam que a atividade de piscicultura é exercida por profissionais com pouca escolaridade. No baixo São Francisco e em Blumenau, Santa Catarina, esse cenário não é diferente, pois os autores expõe que o nível de escolaridade dos envolvidos é baixo (GOMES et al., 2012; RIBEIRO-NETO et al., 2016).

Esses parâmetros de perfil da escolaridade encontrados nas regiões do Brasil também são análogos aos de outros países como, por exemplo, em Tarakanda, Distrito de Mymensingh, região ao qual foi avaliado o perfil de seus piscicultores, onde $37 \%$ dos entrevistados tinham apenas a educação primária e em Bagmara, Distrito de Rajshahi, onde 50\% deles tinham até o ensino primário (ALI et al., 2008, 2010). E no delta Sundarban indiano, descrito por Dubey et al. (2016) os piscicultores estudaram até o oitava ano. Ao ser feito um estudo comparativo das práticas de aquicultura na região de Andhra Pradesh e Bengala Ocidental, evidencia-se que $48 \%$ dos piscicultores estudaram até o ensino primário (JAWAHAR ABRAHAM et al., 2010). A predominância de piscicultores com ensino médio é presente na cidade de Lumindo, Nagaon, Distrito de Assam, descrito por Gupta and Dey (2014) e em dois Distritos de Assam, Darrang e Nagaon, descritos por Goswami et al. (2002).

O nível de escolaridade dos piscicultores em geral vão até o ensino médio, com grande predominância no ensino primário, este panorama pode ser responsável pelo desenvolvimento paulatino das pisciculturas, visto que o acesso ao ensino completo demanda tempo e geralmente as comunidades de Porto Grande são distantes das escolas da sede do município, o que pode ser justificado pela visível frequência de entrevistados com ensino fundamental incompleto. Nesta perspectiva, infere-se que os piscicultores são limitados a adoção de novas tecnologias, aprimoramento do manejo e acesso a financiamentos e programas voltados para criação de peixes. O que prejudica extensamente o desenvolvimento da piscicultura e consequentemente implica no diagnostico do perfil socioeconômico desses criadores de peixes.

\subsection{Estado conjugal}

Quanto ao estado conjugal dos entrevistados, a maioria é casada. Resultados similares são os encontrados no IMEA (2014), onde a maioria, 73,34\% dos piscicultores mato-grossenses são casados. Nakauth et al. (2015), ressaltam que entre os piscicultores entrevistados, a maioria foram casados, e que isso se deve provavelmente a frequência de idade que eles apresentam. Predominância de piscicultores casados também foi observada nos estudos de Araújo (2015) no município de Coremas (PB), onde $100 \%$ eram casados, evidenciando que a atividade de piscicultura na região é praticada por chefes de famílias.

\subsection{Caracterização de bens duráveis e moradia}

No aspecto de bens duráveis foi identificada uma variedade de itens e produtos. São vários os aspectos que se podem levar em consideração para a caracterização da moradia. A estruturação da construção das residências é distribuída em três tipos principais, madeira, alvenaria e mista (alvenaria e madeira), que apresentam revestimentos de quatro categorias, (i) cimento, (ii) barro, (iii) cerâmica, (iv) madeira, (i) casas revestidas de cimento, que é feito um piso queimado de cimento e um pó de 
coloração escolhido, (ii) casas de barro construídas artesanalmente de forma simples, (iii) casas de cerâmica, que se classifica em lajotas simples e porcelanatos e (iv) casas de madeira, para as casas que estão em um nível mais elevados do solo em relações as demais residências são também observadas (Tabela 1).

Quanto a cobertura das residências, divide-se em telha de amianto, cavaco e palha. Ressalta-se, que cavaco é produto de fácil acesso na região e a palha é um material em grande disponibilidade, retirado direto da natureza e fácil manuseio, ambos de baixo custo. As residências, no geral, são próprias, não havendo aluguéis ou cessões, apresentam uma quantidade razoável de cômodos, tais como banheiro, quarto, sala, cozinha, área de serviço e área de laser (Tabela 1).

Quanto ao acesso à água, a maioria das famílias relatou que possuem poço amazonas em suas residências, há existências de utilização de água de igarapés, destes a maioria inclui a utilização de hipoclorito para tratamento da água para o consumo. Grande parcela dos entrevistados não possui sistema de esgoto, onde os dejetos são despejados a céu aberto feito apenas valas para facilitar o despejo e evitar o acúmulo ao redor da residência. O tipo de fossa mais comum existente nas residências é a rudimentar, aquela que se caracteriza pelo buraco feito no solo, muitas vezes sem uma cobertura, sem revestimento de cimento que está representada, a fossa séptica, também foi relatada por uma pequena parcela dos entrevistados, caracterizada por uma estrutura mais beneficiada, que possui revestimento de cimento, com tubulação de esgoto mais propicia.

Quanto ao destino do lixo produzido nas residências, a maioria dos entrevistados apontou que praticam a queima do lixo, pois não há um sistema de coleta regular nas comunidades, e alguns afirmam acumular o lixo em um local próximo da residência.

Em relação a disponibilidade de energia elétrica, os entrevistados afirmaram que o Município de Porto Grande, tem grandes problemas com a falta de energia constante. Essa energia é distribuída pela Companhia de Eletricidade do Amapá- CEA. As residências na comunidade de Munguba são beneficiadas. Para tanto, boa parte delas utilizam o gerador de energia, visto que não há ligação da rede principal para os ramais de suas residências.

Tabela 1- Critérios investigados dos entrevistados quanto à moradia, infraestrutura e bens duráveis no Município de Porto Grande, Amapá, Brasil

\begin{tabular}{|c|c|c|c|}
\hline Eixos & Aspectos & Frequência Absoluta & Frequência Relativa(\%) \\
\hline \multirow{2}{*}{ Estado conjugal } & Solteiro & 5 & 27 \\
\hline & Casado & 13 & 73 \\
\hline \multirow{2}{*}{ Benefício Social } & Sim & 5 & 27 \\
\hline & Não & 13 & 73 \\
\hline \multirow{8}{*}{$\begin{array}{l}\text { Bens duráveis } \\
\text { de maior ocorrência }\end{array}$} & Televisão & 16 & 89 \\
\hline & Fogão & 16 & 89 \\
\hline & Bicicleta & 14 & 78 \\
\hline & Freezer & 13 & 73 \\
\hline & Máquina de lavar roupa & 13 & 73 \\
\hline & Liquidificador & 12 & 67 \\
\hline & Geladeira & 11 & 61 \\
\hline & Ventilador & 9 & 50 \\
\hline \multicolumn{4}{|c|}{ Moradia } \\
\hline \multirow{3}{*}{ Material da construção } & Alvenaria & 5 & 28 \\
\hline & Mista & 3 & 11 \\
\hline & Madeira & 11 & 61 \\
\hline \multirow{3}{*}{ Tipo de Cobertura } & Telha & 16 & 89 \\
\hline & Cavaco & 1 & 6 \\
\hline & Palha & 1 & 6 \\
\hline
\end{tabular}


Tabela 1- Continuação...

\begin{tabular}{|c|c|c|c|}
\hline Eixos & Aspectos & Frequência Absoluta & Frequência Relativa(\%) \\
\hline \multicolumn{4}{|c|}{ Moradia } \\
\hline \multirow{3}{*}{ Tipo de Piso } & Barro & 4 & 22 \\
\hline & Cerâmica & 4 & 22 \\
\hline & Madeira & 3 & 17 \\
\hline \multirow{3}{*}{ Abastecimento de Água } & Poço & 16 & 89 \\
\hline & Igarapé & 2 & 11 \\
\hline & Até 2 cômodos & 3 & 17 \\
\hline \multirow{6}{*}{ Cômodos na residência } & Até 3 cômodos & 5 & 28 \\
\hline & Até 4 cômodos & 4 & 22 \\
\hline & Até 5 cômodos & 2 & 11 \\
\hline & Até 6 cômodos & 3 & 17 \\
\hline & Até 7 cômodos & 1 & 6 \\
\hline & Mais de 7 cômodos & 0 & 0 \\
\hline \multirow{3}{*}{ Tratamento da água } & Hipoclorito & 13 & 72 \\
\hline & Não realiza & 5 & 28 \\
\hline & Não & 11 & 61 \\
\hline \multirow[t]{2}{*}{ Existência de Esgoto } & Sim & 7 & 39 \\
\hline & Fossa rudimentar & 12 & 67 \\
\hline \multirow[t]{2}{*}{ Tipo de fossa } & Fossa séptica & 6 & 33 \\
\hline & Gerador & 2 & 11 \\
\hline \multirow[t]{2}{*}{ Distribuição de Energia } & Energia Elétrica & 16 & 89 \\
\hline & Acumulado & 1 & 6 \\
\hline Destino do Lixo & Queimado & 17 & 94 \\
\hline Estado de Moradia & Própria & 18 & $100 \%$ \\
\hline
\end{tabular}

\subsection{Piscicultura como renda principal da família}

Algumas das famílias entrevistadas relataram receber benefício social (aposentadoria, bolsa família, etc). A renda das famílias entrevistadas varia de $\mathrm{R} \$ 700,00$ a $\mathrm{R} \$ 2.000,00$, neste período o salário mínimo vigente era de $\mathrm{R} \$ 880,00$ (Tabela 2).

Os resultados obtidos para esse item pressupõem que o desenvolvimento das pisciculturas ainda não consegue suprir todas as necessidades das famílias que trabalham nessa atividade, pois grande parcela dos entrevistados necessita trabalhar em uma atividade alternativa para complementação da renda. Os demais têm a piscicultura como renda exclusiva.

No município de Porto Grande, região Norte do Brasil, apenas 39\% dos piscicultores declararam a piscicultura como atividade principal. Ou seja, não atingiram um percentual de 50\%, precisando de outras atividades alternativas para complementação de renda, dentre essas atividades à agricultura. Na mesma região, Sarah et al. (2013) descrevem que grande porcentagem dos produtores não tinham a piscicultura como atividade principal em suas propriedades de Cruzeiro do Sul, no estado do Acre. Este cenário se apresenta em outras regiões.

Na região Nordeste, alguns estudos demonstraram que, para algumas áreas, a piscicultura não é a atividade principal das famílias envolvidas. Estas tem a necessidade de trabalharem, em sua maioria, em atividades agrícolas de subsistência, como por exemplo, no município de Matinha, Maranhão, descrito por Silva (2016); no baixo São Francisco, Sergipe, por Ribeiro-Neto et al. (2016); no baixo São Francisco Alagoano por Araújo e Sá (2008). Na região Sul, descrita nas pequenas propriedades fronteiriça do Sudoeste do Paraná/Brasil por Dutra et al. (2014). Na região Sudeste, apresenta-se no Vale do Ribeira (SP) por Corrêa et al. (2008). Na região Centro-Oeste também constata esse resultado na Bacia do Alto Taquari (MS), por Rotta 
(2003); e no estado de Mato Grosso do Sul, por Dias (2015). A microrregião Cuiabana no estado do Mato Grosso a atividade de piscicultura é descrita como principal renda das famílias (BARROS et al., 2011) o que difere das demais regiões. Em regiões externas ao Brasil é frequente utilização da agricultura como alternativa para complementação da renda, por exemplo, em Tarakanda, Distrito de Mymensingh (ALI et al., 2010) e nos Distritos de Assam (GOSWAMI et al., 2002) e no delta Sundarban indiano (DUBEY et al., 2016).

Tabela 2- Informações econômicas piscicultores entrevistados no Município de Porto Grande, Amapá, Brasil.

\begin{tabular}{cccc}
\hline $\begin{array}{c}\text { Piscicultura- } \\
\text { Principal Renda }\end{array}$ & Sim & 8 & 39 \\
\hline $\begin{array}{c}\text { Atividade } \\
\text { alternativa }\end{array}$ & Não & 11 & 61 \\
\hline $\begin{array}{c}\text { Trabalhos diversos } \\
\text { Rendimento mensal } \\
\text { provido da piscicultura }\end{array}$ & $\mathrm{R} \$ 700,00$ a $\mathrm{R} \$ 1.000,00$ & 10 & 56 \\
& $\mathrm{R} \$ 1.100,00$ a $\mathrm{R} \$ 1.500,00$ & 8 & 54 \\
\hline $\mathrm{R} \$ 1.600,00$ a $\mathrm{R} \$ 2.000,00$ & 3 & 14 \\
& Não respondeu & 2 & 11 \\
\hline
\end{tabular}

\section{Conclusões}

A peculiaridade existente no município de Porto Grande, estado Amapá de possuir potencialidades para o desenvolvimento da piscicultura desde grande disponibilidade de malha hídrica e composta de uma grande variedade de assembleias de peixes que possibilitam o cultivo, colabora para que as famílias introduzam como fonte de renda a criação de peixes. A caracterização do perfil socioeconômico dessas famílias possibilitou um leque de informações que nunca foram discutidas por outros trabalhos para a região, o que enaltece a importância desse levantamento socioeconômico.

Dentre as principais informações geradas estão a renda das famílias não ultrapassam $\mathrm{R} \$ 2.000,00$ reais, a maioria dos piscicultores não possuem o ensino fundamental completo, com predominância do gênero masculino na atividade, a piscicultura não é a renda exclusiva das famílias, necessitando geralmente trabalhar na agricultura ou em atividades diversificadas. Esse perfil gerado pode estar interligado com a necessidade do desenvolvimento da cadeia produtiva da piscicultura na região. Esse fator pode ser minimizado através de formulações de políticas públicas para a área aquícola que se enquadre no perfil das comunidades rurais do município de Porto Grande, Amapá. Gerando um quadro de prospecção da evolução de desenvolvimento local que gere bem-estar e renda para essas famílias para que tenham subsídios que possam contribuir com desenvolvimento regional e econômico. Funcionando essa dinâmica, consequentemente, o perfil socioeconômico dos piscicultores originará mudanças positivas.

\section{Agradecimentos}

À Universidade do Estado do Amapá-UEAP e a Universidade Federal do Amapá-UNIFAP-UNIFAP, por subsidiar as pesquisas de campo. Ao Programa de Pós-Graduação de Mestrado em Desenvolvimento Regional-PPGMDR, por ceder equipamentos e instrumentos. A Secretaria Municipal de Agricultura e Pesca de Porto Grande, Amapá, Brasil-SEMAP, pela contribuição e apoio logístico.

\section{Referências}

AGBOOLA WL. Improving Fish farming Productivity to wards Achieving Food Security in Osun State, Nigeria: A Socioeconomic Analysis. Annals of Biological Research,2011,3:62-74.

ALI MH, AZAD MAK, ANISUZZAMAN M \& CHOWDHURY MMR. HOQUEANd M.I. Shariful Livelihood status of the fishfarmers in some selected areas of Tarakanda upazila of Mymensingh district. Journal Agroforestry Enviroment,2010,2: 85-89. 
ALI MH, HOSSAIN MD, HASANAND ANGM \& BASHAR MA. Assessment of the livelihood status of the fishfarmers in some selectedareas of Bagmaraupazillaunder Rajs hahidistrict. Journal Bangladesh Agricultural University,2008,6: $367-374$.

ARAÚJO JS \& SÁ FP. Sustentabilidade da piscicultura no baixo São Francisco alagoano: condicionantes socioeconômicos. Ambiente e Sociedade,2008, 9: 405-424.

ARAÚJO LS. Estudo da Sustentabilidade da Piscicultura no Município de Coremas PB. Pombal, Pernambuco, [dissertation]. Dissertação de Mestrado em Sistemas Agroindustriais - PPGSA, da Universidade Federal de Campina Grande UFCG/ CCTA. 2015, $62 \mathrm{f}$.

AREAS SM, TRINDADE TC, LIMA AMM, MOURA QL \& ALMEIDA JBA. Dinâmica socioambiental da piscicultura de água doce em tanques rede como alternativa de produção local em ambientes Amazônicos. Revista AgroAmbiente,2014, 8: 277-287.

BARROS, A.F.; MARTINS, M.E.G.; SOUZA, O.M. Caracterização da piscicultura na microrregião da Baixada Cuiabana, Mato Grosso, Brasil. Boletim do Instituto de Pesca,2011, 37(3): 261-273.

BOEGER WA \& BORGHETTI JR. O papel do Poder Público no Desenvolvimento da Aqüicultura Brasileira. In: Ostrensky A, Borghetti JR \& Soto Doris (Org.). Aquicultura no Brasil: o desafio é crescer. Brasília, FAO, 2008,p. 95-114.

CORREAA CF, SCORVO FILHO JD, TACHIBANA L \& LEONARD AFG. Caracterização e situação atual da cadeia de produção da piscicultura do Vale do Ribeira. Informações Econômicas,2008,38: 30-36.

DIAS EF. Governança e licenciamento ambiental para piscicultura: um estudo de multicasos no estado de Mato Grosso do Sul- Dourados. MS: UFGD, 2015,65 p.

DUBEY SK, CHAND BK, TRIVEDI RK, MANDAL B \& ROUT SK. Evaluationontheprevailingaquaculture practices in the Indian Sundarban delta: An insight analysis. Journal of Food, Agriculture \& Environment,2016, 14:133-141.

DUTRA FM, BITTENCOURT F \& FEIDEN A. Perfil aquícola de pequenas propriedades fronteiriça do Sudoeste do Paranál Brasil . Florianópolis, Extensio: Revista Elotrônica de Extensão,2014, 11: 180-189.

ECOTUMUCUMAQUE. Estudo de Impacto Ambiental do Aproveitamento Hidrelétrico Cachoeira Caldeirão.Macapá - AP, 2010,65 p.

GAMA CS. A criação de tilápia no estado do Amapá como fonte de risco ambiental. Acta Amazônica,2008, 38 : 525 - 530.

GIL AC. Como elaborar projetos de pesquisa. São Paulo, Atlas, 120 p,2008.

Gomes G, Kaleski DW, Cunha PR, Toledo F. Avaliação das contribuições do Programa de Fortalecimento da Agricultura Familiar - PRONAF e a melhoria da renda familiar dos piscicultores de Blumenau, Santa Catarina. Gestão \& Regionalidade,2012, 28: 21-31.

GOSWAMI M, SATHIADHAS R, GOSWAMI UC \& OJHA SN. Socio-economic dimension of fishfarmingin Assam. Journal of the Indian Fisheries Association ,2002, 29: 103-110.

GUPTA T \& DEY M. Socioeconomic and cultural profile of fishfarmers: a study in and around Lumding town, Nagaon Districtof Assam. International Journal of Life scienceand Pharma Research,2014, 14: 83-93.

INSTITUTO BRASILEIRO DE GEOGRAFIA E ESTATÍSTICA - IBGE. [Internet]. Censo Demográfico. IBGE. Brasília: DF, 2010. Disponível em: http://www.ibge.gov.br/censo2010/primeiros_dados_. Acessado em 20 de junho de 2016.

INSTITUTO BRASILEIRO DE GEOGRAFIA E ESTATÍSTICA - IBGE (2012) Estudos e Pesquisas Informação Demográfica e Socioeconômica, número 29: Síntese de Indicadores Sociais Uma análise das condições de vida da população brasileira. 293 páginas. Rio de Janeiro,. 
Federação da Agricultura e Pecuária do Estado do Mato Grosso, CUIABÁ,2014,103f.

JAWAHAR AT, SIL SK \& VINEETHA P. A comparative study of the aquaculture practices adopted by fishfarmers in Andhra Pradesh and West Bengal. Indian Journal Fish.,2010, 57: 41-48.

NAKAUTH ACS, NAKAUTH RF \& NÓVOA NAC. Caracterização da Piscicultura no Município de Tabatinga-AM. Igapó:Revista de Educação, Ciência e Tecnologia do Ifam,2015, 9: 54-64.

PPCDAP. Plano de Prevenção e Controle do Desmatamento e Queimadas do Estado do Amapá - Contexto e Ações. Secretaria Especial de Desenvolvimento Econômico e Secretaria de Estado do Meio Ambiente, Governo do Estado do Amapá, Macapá,2009, $120 f$.

ROTTA MA. Diagnóstico da piscicultura na Bacia do Alto Taquari - MS. Corumbá: Embrapa Pantanal, Boletim de Pesquisa e Desenvolvimento/Embrapa Pantanal,2003,31p.

SARAH MGM, SANTOS MIS \& SANTIAGO ACC. Aspectos da atividade de piscicultura praticada por produtores rurais no Município de Cruzeiro do Sul - Acre. Enciclopédia Biosfera,2013, 16: 568-586.

SOUZA JÁ. Estudo de impactos sociais, econômicos e ambientais, ocasionados pela piscicultura em tanques-rede na região de Paulo Afonso-BA. Dissertação de Mestrado em Ciências do Ambiente- Universidade Federal do Tocantins, [dissertation]. Palmas. 114 f, 2006. Disponível em: <http://download.uft.edu.br/?d=54579acb-4544-40b6-8f2d-63670c112eb1; 1.0: joaoAugusto2006.pdf> Acessado: 19 julho de 2017.

SUDAM. Projeto de Hidrologia e Climatologia da Amazônia. Atlas climatológico da Amazônia Brasileira. Belém: SUDAM,1984,125p.

Netiê Izabel da Silva de Oliveira

Centro Integrado de Formação Profissional em Pesca e Aquicultura do Amapá - CIFPA, Macapá - AP, Brasil.

Email: netieoliveira@gmail.com

Participação do autor: Coleta e análise dos dados, Redigiu o trabalho

\section{Alexandro Cezar Florentino}

Universidade Federal do Amapá, Macapá - AP, Brasil.

Email: alexandrocezar@unifap.br

Participação do autor: contribuições substanciais para a concepção do trabalho; Orientou e realizou revisão substancial. 